Pacific Journal of Mathematic 


\title{
FORMS OF THE AFFINE LINE AND ITS ADDITIVE GROUP
}

\author{
Peter Russell
}

\begin{abstract}
Let $k$ be a field, $X_{0}$ an object (e.g., scheme, group scheme) defined over $k$. An object $X$ of the same type and isomorphic to $X_{0}$ over some field $K \supset k$ is called a form of $X_{0}$. If $k$ is not perfect, both the affine line $A^{1}$ and its additive group $G_{a}$ have nontrivial sets of forms, and these are investigated here. Equivalently, one is interested in $k$-algebras $R$ such that $K \otimes_{k} R \cong K[t]$ (the polynomial ring in one variable) for some field $K \supset k$, where, in the case of forms of $\mathbf{G}_{a}, R$ has a group (or co-algebra) structure $s: R \rightarrow R \otimes_{k} R$ such that $(K \otimes s)(t)=$ $t \otimes 1+1 \otimes t$. A complete classification of forms of $\mathbf{G}_{a}$ and their principal homogeneous spaces is given and the behaviour of the set of forms under base field extension is studied.
\end{abstract}

If $k$ is perfect, all forms of $\mathbf{A}^{1}$ and $\mathbf{G}_{a}$ are trivial, as is well known (cf. 1.1). So assume $k$ is not perfect of characteristic $p>0$. Then a nontrivial example (cf. [5], p. 46) of a form of $\mathbf{G}_{a}$ is the subgroup of $\mathbf{G}_{a}^{2}=\operatorname{Spec} k[x, y]$ defined by $y^{p}=x+a x^{p}$ where $a \in k, a \notin k^{p}$. We show that this example is quite typical (cf. 2.1): Every form of $\mathbf{G}_{a}$ is isomorphic to a subgroup of $\mathbf{G}_{a}^{2}$ defined by an equation $y^{p^{n}}=a_{0} x+$ $a_{1} x^{p}+\cdots+a_{m} x^{p^{m}}, a_{i} \in k, a_{0} \neq 0$. Analyzing the equivalence relation induced on the right hand side polynomials by isomorphism of the groups which they define, we obtain a description of the set of forms of $\mathbf{G}_{a}$ split by $k^{p^{-n}}$ as, essentially, the quotient of an infinite direct sum of copies of $k / k^{p n}$ under a certain group action (cf. 2.5).

If $G$ is a nontrivial form of $\mathbf{G}_{a}$, we show that $\operatorname{End}_{k} G$ is a finite field (cf. 3.1). This allows one to compute the set of $k_{s} / k$-forms of $G$ ( $k_{s}$ a separable algebraic closure of $k$ ) using Golois cohomology. This set is nontrivial in general, in contrast to the same situation for $\mathbf{G}_{\alpha}$.

A form $X$ of $\mathbf{A}^{1}$ may fail to have a group structure for two reasons. First, and this is the serious failure, $X_{k_{s}}$ may not have enough (i.e., infinitely many) automorphisms. As an example, with the identity as the only automorphism, one may take $\mathbf{P}^{1}-\{q\}$, where $\mathbf{P}^{1}$ is the projective line and $q$ is a purely inseparable point of degree $p^{n}>2$. The general case here seems to be rather complex. Secondly, $X_{k_{s}}$ may have enough automorphisms, but $X$ may not have a rational point. We show that then $X$ is a principal homogeneous space for a form of $\mathbf{G}_{a}$ (cf. 4.1). This gives a new interpretation of a result of Rosenlicht ([4], p. 10, theorem) on curves with exceptionally many automorphisms (cf. 4.2). 
1. Throughout this paper $k$ will be a fixed base field, $\bar{k}$ an algebraic closure of $k, k_{i}=k^{p^{-\infty}}(p=$ char $k)$ the perfect and $k_{s}$ the separable closure of $k$ in $\bar{k}$. Reference to $k$ will usually be omitted.

It is well known (cf. [5], p. 34 and [6], p. 108) that a form $G$ of $\mathbf{G}_{a}$ is split by $k_{i}$, that is, $G_{k_{i}} \cong \mathbf{G}_{a k_{i}}$. The same is true for forms $X$ of $\mathbf{A}^{1}$. For the sake of completeness, and to establish some notation, we briefly outline the argument. The idea is to investigate the complete regular curve $P$ determined by $X$. As a matter of terminology, we call a scheme $Y$ regular if all its local rings are regular, and nonsingular if $Y_{K}$ is regular for any $K \supset k$. As is well known, $Y_{K}$ nonsingular implies $Y$ nonsingular, and $Y$ is nonsingular if and only if $Y_{k p^{-1}}$ is regular. The existence of forms of $\mathbf{A}^{1}$ is closely connected with the divergence of these notions if $k$ is not perfect. If $Y$ is a curve, we denote by $\widetilde{Y}_{K}$ the regular curve obtained by normalizing $Y_{K}$.

Lemma 1.1. Let $X$ be a form of $\mathbf{A}^{1}$ and $P \supset X$ a complete regular curve.

(i) $P-X$ is a point purely inseparable over $k$.

(ii) There is a unique minimal field $k^{\prime} \supset k$ such that $X_{k^{\prime}} \cong \mathbf{A}_{k^{\prime}}^{1}$, and $k^{\prime}$ is purely inseparable of finite degree over $k$.

Proof. The genus of $\widetilde{P}_{k_{i}}$ is zero since this is so after suitable base field extension and since, $k_{i}$ being perfect, the genus does not change under base field extension (cf. [1], V, 5 , Th. 5). Since $\widetilde{P}_{\vec{k}}$ has a rational point, $\widetilde{P}_{\bar{k}} \cong \mathbf{P}_{\bar{k}}^{1}$. An open subscheme of $\mathbf{P}_{K}^{1}$ ( $K$ any field) is a form of $\mathbf{A}_{K}^{1}$ if and only if it is the complement of a purely in separable point. Hence $\widetilde{P}_{\bar{k}}-X_{\vec{k}}$ is a point, and a fortiori $P-X$ (resp. $P_{k_{i}}-X_{k_{i}}$ ) is a point purely inseparable over $k$ (resp. rational over $k_{i}$ ). In particular, $\widetilde{P}_{k_{i}} \cong \mathbf{P}_{k_{i}}^{1}$ and $X_{k_{i}} \cong \mathbf{A}_{k_{i}}^{1}$. If $K \supset k$ is any field such that $X_{K} \cong \mathbf{A}_{K}^{1}$, then $\widetilde{P}_{K}-X_{K}$ is a point rational over $K$ and $K$ contains (up to unique isomorphism) the residue field $k_{1}$ of $P-X$. Now pass to $X_{k_{1}}$ and continue this process. After finitely many steps, we reach a field $k^{\prime} \subset K, k \subset k^{\prime} \subset k_{i}$, such that $\widetilde{P}_{k^{\prime}} \cong \mathbf{P}_{k^{\prime}}$, and $\widetilde{P}_{k^{\prime}}-X_{k^{\prime}}$ is rational over $k^{\prime}$. Then $X_{k^{\prime}} \cong \mathbf{A}_{k^{\prime}}^{1}$.

$\mathbf{A}^{1}=$ Spec $k[t]$ admits, up to choice of origin, a unique group structure (given by $s(t)=t \otimes 1+1 \otimes t$ if the origin is at $t=0$ ), and any automorphism of $\mathbf{A}^{1}$ sending the origin to the origin is a group homomorphism. Let $G$ and $G^{\prime}$ be groups with origins $q$ and $q^{\prime}$ and $\psi$ an isomorphism of the underlying schemes, supposed to be forms of $\mathbf{A}^{1}$, such that $\psi(q)=q^{\prime}$. Then $\psi$ is a homomorphism of groups after base field extension, which means that a certain diagram of morphisms (over $k$ ) commutes after base extension and so is commutative to begin with. Hence $\psi$ is an isomorphism of groups. This gives: 
Lemma 1.2. Let $X$ be a form of $\mathbf{A}^{1}$. Then any group scheme $G$ with underlying scheme $X$ is a form of $\mathbf{G}_{a}$. The group structure (if it exists) is unique up to choice of origin. If $X_{K} \cong \mathbf{A}_{K}^{1}$, then $G_{K} \cong \mathbf{G}_{a K}$.

We assume from now on that char $k=p>0$. We denote by $\Theta^{n}$ the base change functor deduced from

$$
\begin{aligned}
\varphi^{n}: k & \longrightarrow k \\
a & \longmapsto a^{p^{n}} .
\end{aligned}
$$

For any scheme $X$ there is a canonical morphism $F_{X}^{n}: X \rightarrow \Theta^{n} X$. If $X$ is a group scheme, so is $\Theta^{n} X$ and $F_{X}^{n}$ is a homomorphism. Referring to [3], p. I. 1-5 for more details, we remark only that if $X=\operatorname{Spec} R$ is affine, then $\Theta^{n} X=\operatorname{Spec}\left(\left(k, \phi^{n}\right) \bigotimes_{k} R\right)$ where $\left(k, \phi^{n}\right)=k$ considered as a right $k$-algebra via $\varphi^{n}$ and as a left $k$-algebra in the usual way, and that $F_{X}^{n}$ is deduced from

$$
\begin{aligned}
F_{R}^{n}:\left(k, \varphi^{n}\right) \bigotimes_{k} R & \longrightarrow R \\
a \otimes x & \longmapsto a x^{p^{n}} .
\end{aligned}
$$

$\Theta^{n}$ accomplishes, up to isomorphism, the same as the base change $k \subset k^{p^{-n}}$. More precisely, if $K$ is purely inseparable of exponet $\leqq n$ over $k$ (that is, $K^{p^{n}} \subset k$ ), there is a commutative diagram

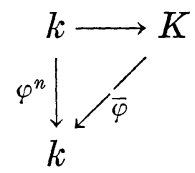

and we have $\Theta^{n} X \cong(k, \bar{\varphi}) \otimes_{K} X_{K}$ for any scheme $X$ over $k$.

Lemma 1.3. Let $X$ be a form of $\mathbf{A}^{1}$. For any integer $n \geqq 0, F_{X}^{n}$ is a purely inseparable morphism of degree $p^{n}$. For any morphism $\psi: X \rightarrow Y$ of finite degree, there is a unique factorization $\psi=\bar{\psi} F_{X}^{m}$ where $p^{m}$ is the inseparable degree of $\psi$ and $\bar{\psi}$ is a separable morphism. Finally, there is an integer $n \geqq 0$ such that $\Theta^{n} X \cong \mathbf{A}^{1}$.

Proof. The last statement follows from 1.1 and the remark above. The function field $\kappa(X)$ of $X$ is separable of transcendence degree one over $k$ and so has, for each $n$, a unique subfield $\supset k$ over which it is purely inseparable of degree $p^{n}$, namely

$$
k\left(\kappa(X)^{p^{n}}\right) \cong\left(k, \varphi^{n}\right) \underset{k}{\bigotimes} \kappa(X)=\kappa\left(\Theta^{n} X\right)
$$

(cf. [2], p. 186, Th. 19 and p. 179, corollary). This proves the first statement and the second follows in view of the fact that $\Theta^{m} X$ is normal. 
1.4. Let $X$ be a form of $\mathbf{A}^{1}$. We let $n(X)$ be the least $n$ such that $\Theta^{n} X \cong \mathbf{A}^{1}$ or, equivalently, the least $n$ such that $X$ has a splitting field of exponent $n$ over $k$.

The point of 1.3 is that the affine ring $R$ of $X$ has a unique maximal subring of the form $S=k[x]$ such that $R^{p^{n}} \subset S$ for som $n$, and that the only other subrings with this property are the rings $k\left[x^{p^{m}}\right], m \geqq 0$. Note, however, that $n(X)$ need not be the least $n$ such that $\kappa\left(\Theta^{n} X\right) \cong k(t)$ or, equivalently, that $\Theta^{n} X \subset \mathbf{P}^{1} . \quad Y=\mathbf{P}^{1}-\{q\}, q$ purely inseparable and not rational over $k$, is one example and, giving $Y$ some further twist, one can find $X$ such that $\Theta^{n} X \cong Y$ and $n>1$.

2. Since $\mathbf{G}_{a}$ is defined over the prime field, we may identify $\mathbf{G}_{a}$ and $\Theta \mathbf{G}_{a}$. Then $F=F_{\mathbf{G}_{a}} \in A=\operatorname{Hom}_{k}\left(\mathbf{G}_{a}, \mathbf{G}_{a}\right)$. It is well known that $A=k[F]$, a ring of noncommutative polynomials with relations $F a=a^{p} F$ for $a \in k$. We define the power series ring $\hat{A}=k[[F]]$ in the same way. Let $\varepsilon: A \rightarrow k$ be the natural augmentation. We let $A^{*}=\varepsilon^{-1}\left(k^{*}\right)$ and $A^{* *}=\varepsilon^{-1}(1)$ and make corresponding definitions for $\hat{A}$. As in the case of ordinary power series, $\hat{A}^{*}$ is the group of units of $\hat{A}$. By truncation we obtain groups $U_{n}=\hat{A}^{*} / \hat{A} F^{n} \cong A^{*} / A F^{n}$. $\tau=\sum_{i=0}^{m} a_{i} F^{i} \in A, a_{m} \neq 0$, has degree $p^{m}$ as a morphism $\tau: \mathbf{G}_{a} \rightarrow \mathbf{G}_{a}$, and we also give it degree $p^{m}$ in the graded ring $k[F]$. Note that $A^{*} \subset A$ is the subset of separable homomorphisms. An endomorphism $\lambda: k \rightarrow k$ commutes with $p$-th powers and so extends to an endomorphism $\lambda: A \rightarrow A$

$$
\sum a_{i} F^{i} \longmapsto \sum \lambda\left(a_{i}\right) F^{i}
$$

In the particular case $\lambda=\phi^{n}$ we put $\lambda(\tau)=\tau^{(n)}$ for $\tau \in A$ and $\lambda(A)=$ $A^{(n)}$. $\tau^{(n)}$ is characterized by $F^{n} \tau=\tau^{(n)} F^{n}$.

If $G=\operatorname{Spec} R$ is an affine group with group operation $s: R \rightarrow$ $R \otimes_{k} R, \operatorname{Hom}_{k}\left(G, \mathbf{G}_{a}\right)$ may be identified with

$$
\{r \mid s(r)=r \otimes 1+1 \otimes r\} \subset R \cong \operatorname{Hom}(k[t], R) .
$$

In particular, $A$ is identified with the set of $p$-polynomials

$$
f(t)=a_{0} t+a_{1} t^{p}+\cdots+a_{m} t^{p^{m}} \in k[t] \text {. }
$$

THEOREM 2.1. Let $G$ be a form of $\mathbf{G}_{a}$. Then $G$ is isomorphic to a subgroup Spec $k[x, y] / I$ of $\mathbf{G}_{a}^{2}=\operatorname{Spec} k[x, y]$ where $I$ is generated by a polynomial $y^{p^{n}}-\left(a_{0} x+a_{1} x^{p}+\cdots+a_{m} x^{p^{m}}\right), a_{0} \neq 0$. Equivalently, $G$ is a fiber product 


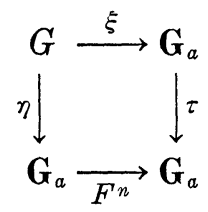

where $\tau=a_{0}+a_{1} F+\cdots+a_{m} F^{m} \in A^{*}$. Conversely, any $G$ defined that way is a form of $\mathbf{G}_{a}$.

Proof. Let $G=\operatorname{Spec} R, s: R \rightarrow R \bigotimes_{k} R$ the group operation,

$$
\bar{s}:\left(k, \varphi^{n}\right) \bigotimes_{k} R \longrightarrow\left(k, \varphi^{n}\right) \bigotimes_{k} R \bigotimes_{k} R \cong\left(\left(k, \varphi^{n}\right) \bigotimes_{k} R\right) \bigotimes_{k}\left(\left(k, \varphi^{n}\right) \bigotimes_{k} R\right)
$$

the induced group operation for $\Theta^{n} G$. By 1.3, we have $\Theta^{n} G \cong \mathbf{G}_{a}$ for some $n$, so that $\left(k, \varphi^{n}\right) \bigotimes_{k} R \cong k[t]$ where we can choose $t$ such that $\bar{s}(t)=t \otimes 1+1 \otimes t$. Write $t=\sum a_{i} \otimes y_{i}$ with $a_{i} \in k$ and $y_{i} \in R$. Then

$$
\begin{aligned}
\bar{s}(t)=t \otimes 1+1 \otimes t & =\sum a_{i} \otimes y_{i} \otimes 1+\sum a_{i} \otimes 1 \otimes y_{i} \\
& =\sum \bar{s}\left(a_{i} \otimes y_{i}\right)=\sum a_{i} \otimes s\left(y_{i}\right) .
\end{aligned}
$$

If we choose the $a_{i}$ linearly independent in $k$ considered as a vector space over $k$ via $\varphi^{n}$, i.e., linearly independent over $k^{p^{n}}$, this implies $s\left(y_{i}\right)=y_{i} \otimes 1+1 \otimes y_{i}$. Hence the $y_{i}\left(1 \otimes y_{i}\right)$ define homomorphisms $\eta_{i}: G \rightarrow \mathbf{G}_{a}\left(\Theta^{n} \eta_{i}: \mathbf{G}_{a} \rightarrow \mathbf{G}_{a}\right)$. As observed above, this implies $1 \otimes y_{i}=f_{i}(t)$ where $f_{i}$ is a $p$-polynomial. Applying $F_{R}^{n}$ and putting $x=F_{R}^{n}(t)$, we obtain $y_{i}^{p^{n}}=f_{i}(x)$. Clearly the $y_{i}$ generate $R$ over $k$ and one of them, call it $y$, is a separating variable for $\kappa(G)$. Then $y^{p^{n}}=f(x)=a_{0} x+a_{1} x^{p}+$ $\cdots+a_{m} x^{p^{m}}$, with $a_{0} \neq 0$ since $x$ is separable over $k(y)$. This shows that $k[x, y] \subset R$ is integrally closed. $\kappa(G)$ is separable and purely inseparable over $k(x, y)$, so $k(x, y)=\kappa(G)$ and $R=k[x, y]$. This proves the first statement. The next follows letting $\eta$ be the homomorphism corresponding to $y$ and $\xi=F_{G}^{n}$ the homomorphism corresponding to $x$. Finally, let $R=k[x, y]$ where $y^{p^{n}}=f(x)$. Then $s: R \rightarrow R \bigotimes_{k} R, s(x)=$ $x \otimes 1+1 \otimes x, s(y)=y \otimes 1+1 \otimes y$, is well defined and gives a group structure on $R$. Taking $a_{0}=1$ for simplicity, we have

$$
1 \otimes x=\left(1 \otimes y^{p^{n-1}}-\left(a_{1}^{p^{n-1}} \otimes x+\cdots+a_{m}^{p^{n-1}} \otimes x^{p^{m-1}}\right)\right)^{p}=t_{1}^{p}
$$

in $\left(k, \varphi^{n}\right) \bigotimes_{k} R$. Replacing $1 \otimes x$ by $t_{1}^{p}$ on the right hand side and continuing that way, we find $t \in\left(k, \varphi^{n}\right) \bigotimes_{k} R$ such that $1 \otimes x=t^{p^{n}}$ and $1 \otimes y^{p^{n}}=(f(t))^{p^{n}}$. Spec $R$ is nonsingular, so $\left(k, \varphi^{n}\right) \bigotimes_{k} R$ is reduced. Hence $1 \otimes y=f(t)$, showing that $\left(k, \varphi^{n}\right) \bigotimes_{k} R=k[t]$.

2.2. We write $G=\left(F^{n}, \tau\right)$ (with $\tau \in A^{*}$ ) for a fiber product as in the theorem. Note that $G$ can be so written if and only if $\Theta^{n} G \cong \mathbf{G}_{a}$. 
Proposition 2.3. Let $G=\left(F^{n}, \tau\right), G_{1}=\left(F^{n_{1}}, \tau_{1}\right)$ and assume $n_{1} \leqq n$. Then $G \cong G_{1}$ if and only if there exist elements $\rho \in A^{*}, \sigma \in A$ and $c \in k^{*}$ such that

$$
\tau_{1}^{\left(n-n_{1}\right)}=\left(\rho^{(n)} \tau+F^{n} \sigma\right) c^{-1} .
$$

$\rho$ may be chosen of degree $\leqq p^{n-1}$.

Proof. The monomorphism $(\xi, \eta): G \rightarrow \mathbf{G}_{a}^{2}$ induces an epimorphism of $A$-modules $A \oplus A=\operatorname{Hom}_{k}\left(\mathbf{G}_{a}^{2}, \mathbf{G}_{a}\right) \rightarrow \operatorname{Hom}_{k}\left(G, \mathbf{G}_{a}\right.$ ) (cf. [6], p. 102, proposition). Hence $\operatorname{Hom}_{k}\left(G, \mathbf{G}_{a}\right)=A \eta+A \xi$ with $F^{n} \eta=\tau \xi$ as a defining relation. Since $G$ is reduced and irreducible, $\operatorname{Hom}_{k}\left(G, \mathbf{G}_{a}\right)$ is torsion free.

Let $\psi: G \rightarrow G_{1}$ be an isomorphism and consider the commutative diagram

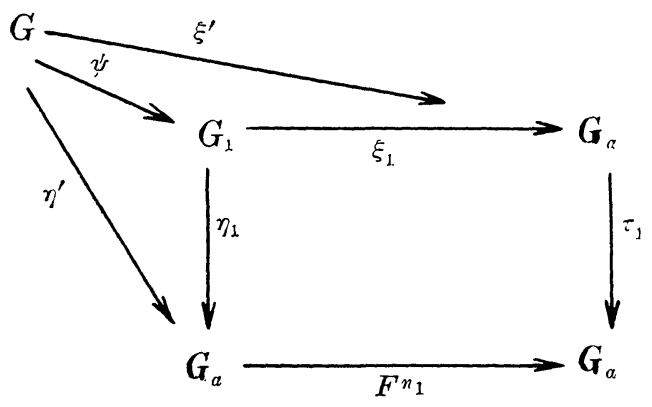

Now $\eta^{\prime}=\eta_{1} \psi=\rho \eta+\sigma \xi$ for some $\rho, \sigma \in A$, and we must have $\rho \in A^{*}$ since $\eta^{\prime}$ is separable. Also, if $\rho=\rho_{1}+\rho_{2} F^{n}$, then $\rho \eta=\rho_{1} \eta+\rho_{2} \tau \xi$. So we can choose $\rho$ of degree $<p^{n}$. Assume first $n=n_{1}$. Then $\xi^{\prime}=$ $\xi_{1} \psi$ is purely inseparable of degree $p^{n}$. By $1.3, \xi_{1} \psi=c \xi$ with $c \in A$ a separable and purely inseparable homomorphism, that is, $c \in k^{*}$. Now

$$
\begin{aligned}
\tau_{1} \xi_{1} \psi=F^{n} \eta_{1} \psi & =F^{n} \rho \eta+F^{n} \sigma \xi=\rho^{(n)} F^{n} \eta+F^{n} \sigma \xi \\
& =\left(\rho^{(n)} \tau+F^{n} \sigma\right) \xi=\left(\rho^{(n)} \tau+F^{n} \sigma\right) c^{-1} \xi_{1} \psi,
\end{aligned}
$$

giving $\tau_{1}=\left(\rho^{(n)} \tau+F^{n} \sigma\right) c^{-1}$. Conversely, define $\xi^{\prime}, \eta^{\prime} \in \operatorname{Hom}\left(G, \mathbf{G}_{a}\right)$ by $\xi^{\prime}=c \xi$ and $\eta^{\prime}=\rho \eta+\sigma \xi$. Then $F^{n} \eta^{\prime}=\tau_{1} \xi^{\prime}$, and we obtain a homomor phism $\psi: G \rightarrow G_{1}$ such that $\xi^{\prime}=\xi_{1} \psi$ and $\eta^{\prime}=\eta_{1} \psi$. Now $\rho$ is invertible in $\hat{A}$ and we can write $\rho^{-1}=\rho_{1}+\sigma_{2} F^{n}$ with $\rho_{1} \in A^{*}$. Then $\tau=$ $\left(\rho_{1}^{(n)} \tau_{1}+F^{n} \sigma_{1}\right) c$ with $\sigma_{1}=\left(\sigma_{2} \rho^{(n)} \tau-\rho_{1} \sigma\right) c^{-1} \in A$. Reversing the roles of $G$ and $G_{1}$ we get $\psi_{1}: G_{1} \rightarrow G$ inverting $\psi$.

Suppose now $n-n_{1}=n_{2} \geqq 0$. In the commutative diagram

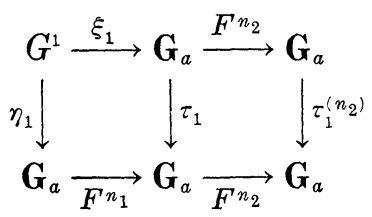


both the left and right square are cartesian. So the big square is cartesian, and consequently $G_{1}=\left(F^{n}, \tau_{1}^{\left(n_{2}\right)}\right)$. Now the previous argument applies.

Since $\left(F^{n}, \tau\right) \cong\left(F^{n}, \tau \varepsilon(\tau)^{-1}\right)$, any $G$ can be written with $\tau \in A^{* *}$. This normalizes $\tau$ to some extent:

COROLlary 2.3.1. Let $G=\left(F^{n}, \tau\right)$. Then $G \cong \mathbf{G}_{a}$ if and only if $\tau c \in A^{(n)}$ for some $c \in k^{*}$. If $\tau=1+a_{1} F+\cdots+a_{m} F^{m} \in A^{* *}$, then $k^{\prime}=k\left(a_{1}^{p^{-n}}, \cdots, a_{m}^{p^{-n}}\right)$ is the minimal splitting field for $G$.

Proof. Since $\mathbf{G}_{a}=\left(F^{n}, 1\right)$, the proposition gives $\tau c=\rho^{(n)}+F^{n} \sigma \in A^{(n)}$ if $G \cong \mathbf{G}_{a}$. Conversely, let $\tau c=\tau_{1}^{(n)}$. Then $\tau_{1} \in A^{*}$ and we can write $1=$ $\rho \tau_{1}+\sigma F^{n}$. So $1=\left(\rho^{(n)} \tau+F^{(n)} \sigma c^{-1}\right) c$ and $\left(F^{n}, 1\right) \cong\left(F^{n}, \tau\right)$. This proves the first statement, and the second follows since we can take $c=1$ above if $\tau \in A^{* *}$.

Corollary 2.3.2. Let $G=\left(F^{n}, \tau\right)$ and $0 \leqq m \leqq n$. Then

$$
\Theta^{m} G=\left(F^{n-m}, \tau\right) \text {. }
$$

Proof. Apply $\Theta^{m}$ to the cartesian square defining G. Noting that $\Theta^{m} \tau=\tau^{(m)}$, we get $\theta^{m} G=\left(F^{n}, \tau^{(m)}\right) \cong\left(F^{n-m}, \tau\right)$.

2.4. For any field $K \supset k$, we define $E(K)$ as the set of isomorphism classes of forms of $\mathbf{G}_{a K}$ and put $E(K, n)=\left\{G \in E(K) \mid \Theta^{n} G \cong \mathbf{G}_{a K}\right\}$.

The rule $(\rho, \sigma, c) \cdot \tau=\left(\rho^{(n)} \tau+F^{n} \sigma\right) c^{-1}$ defines an action of

$$
A^{*} \times A \times k^{*},
$$

endowed with a suitable semi-direct product structure, on $A^{*}$, and 2.3 states that $E(k, n)$ may be considered as the quotient of $A^{*}$ under this action. $A^{*}$ is not a group, but this inconvenience can be avoided by dividing out by $A$ first and passing to the group $U_{n}=A^{*} / A F^{n}$. Let $V_{n}=U_{n} \times k^{*}$. Then the map

$$
\begin{aligned}
V_{n} \times A^{*} / F^{n} A & \longrightarrow A^{*} / F^{n} A \\
(\bar{\rho}, c) \times \bar{\tau} & \longmapsto\left(\rho^{(n)} \tau c^{-1}\right)^{-}
\end{aligned}
$$

(where - denotes taking residue classes) is well defined and gives an action of $V_{n}$ on $A^{*} / F^{n} A$. Clearly all the operations involved are compatible with base field extension. Now 2.3 implies:

Theorem 2.5. The map

$$
\begin{aligned}
A^{*} & \longrightarrow E(k, n) \\
\tau & \longmapsto\left(F^{n}, \tau\right)
\end{aligned}
$$


induces a bijection between the quotient of $A^{*} / F^{n} A$ by the action (*) defined above and $E(k, n)$. This identification is compatible with base field extension.

Similarly, we can define an action

$$
U_{n} \times A^{* *} / F^{n} A \longrightarrow A^{* *} / F^{n} A \text { by } \bar{\rho} \cdot \bar{\tau}=\left(\rho^{(n)} \tau \varepsilon(\rho)^{-p^{n}}\right)^{-} .
$$

Since any $G$ can be written as $G=\left(F^{n}, \tau\right)$ with $\tau \in A^{* *}$, the quotient may again be identified with $E(k, n)$. As an example, let us work out the case $n=1$. Choose a complementary subspace $W_{0}$ for $k^{p}$ in $k$ and for each $i \geqq 1$ let $W_{i}$ be a copy of $W_{0}$. Then $U_{1}=k^{*}$ acts on $W=$ $\bigoplus_{i=1}^{\infty} W_{i}$ by $c \cdot \sum a_{i}=\sum c^{p\left(1-p^{i}\right)} a_{i}$. Letting $\left(F, 1+\sum a_{i} F^{i}\right)$ correspond to the class of $\sum a_{i}$, one identifies $E(k, 1)$ and $W / k^{*}$.

Let $A^{*} / F^{n+1} A \rightarrow A^{*} / F^{n} A$ be the natural map and define $V_{n+1} \rightarrow$ $V_{n}$ by $\left.\left.\overline{(\rho}, c\right) \mapsto \overline{\left(\rho^{(1)}\right.}, c\right)$. Then

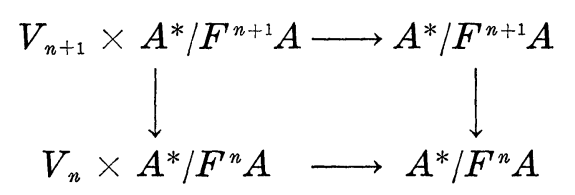

commutes and it follows from 2.3.2 that the induced map on the quotients is $\theta: E(k, n+1) \rightarrow E(k, n)$. Unfortunately there does not seem to be a coherent way to reverse the vertical arrows in order to obtain the inclusion $E(k, n) \subset E(k, n+1)$.

Proposition 2.6. Let $K \supset k$ be a field and

$$
\begin{aligned}
\Psi: E(k) & \longrightarrow E(K) \\
G & \longmapsto G_{K}
\end{aligned}
$$

the natural map.

(i) If $K$ is purely inseparable over $k$, then $\Psi$ is surjective.

(ii) If $k$ is algebraically closed in $K$ and $K$ is separable over $k$, then $\Psi$ is injective.

Proof. (i ) Let $G=\left(F^{n}, \tau\right) \in E(K), \tau=1+a_{1} F+\cdots+a_{m} F^{m}$. There is an integer $r \geqq 0$ such that $a_{i}^{p^{r}}=\alpha_{i} \in k, i=1, \cdots, m$. Let $\tau^{\prime}=1+\alpha_{1} F+\cdots+\alpha_{m} F^{m}$ and $G^{\prime}=\left(F^{n+r}, \tau^{\prime}\right) \in E(k)$. Then $\tau^{\prime}=\tau^{(r)}$ over $K$ and 2.3 implies $G_{K}^{\prime}=\left(F^{n+r}, \tau^{(r)}\right) \cong\left(F^{n}, \tau\right)=G$.

(ii) Let $G=\left(F^{n}, \tau\right), \tau=1+\sum a_{i} F^{i} \in A, \rho=\sum x_{i} F^{i} \in A_{K}^{*}$ with $x_{i}=0$ for $i \geqq n$, and $\sigma=\sum y_{i} F^{i} \in A_{K}$. Suppose $\left(\rho^{(n)} \tau+F^{n} \sigma\right) x_{0}^{-p^{n}}=$ $1+\sum b_{i} F^{i}=\tau^{\prime} \in A$, that is,

$$
\left(\sum_{j=0}^{i-1} x_{j}^{p^{n}} a_{i-j}^{p^{j}}+x_{i}^{p^{n}}+y_{i-n}^{p^{n}}\right) x_{0}^{-p^{n+i}}=b_{i} \in k
$$


for $i \geqq 1$. (Set $y_{i}=0$ for $i<0$ ). We have to show that the same can be done with $x_{i}, y_{i} \in k$. We may clearly assume $G \not \mathbf{G}_{a}$. Then not all $a_{i} \in k^{p^{n}}$ and there is an $r \geqq 1$ such that $a_{1}, \cdots, a_{r-1} \in k^{p^{n}}$ but $a_{r} \notin k^{p^{n}}$. If $r>1$, we can replace $\tau$ by $\left(1-a_{1} F\right) \tau$ (since $a_{1} \in k^{p^{n}}$ ) which has a zero linear term. By an obvious induction argument, we can assume $a_{1}=\cdots=a_{r-1}=0$. Then $(*)$ gives (for $i=r$ )

$$
a_{r} x_{0}^{p^{n}-p^{n+r}}+x_{r}^{p^{n}} x_{0}^{-p^{n+r}}+y_{r-n}^{p^{n}} x_{0}^{-p^{n+r}}=b_{r} .
$$

Put $u=x_{0}^{-1}, v=x_{r} x_{0}^{-p^{r}}$ if $r<n$ (and so $y_{r-n}=0$ ), and $v=y_{n-r} x_{0}^{-p^{r}}$ if $r \geqq n$ (and so $x_{r}=0$ ). In both cases $a_{r} u^{\left(p^{r}-1\right) p^{n}}+v^{p^{n}}=b_{r}$. Extracting $p$-th roots in $k$ from $a_{r}$ and $b_{r}$ as far as possible, we can write $a u^{\left(p^{r}-1\right) p^{n_{1}}}+v^{p^{n_{1}}}=b$ where not both $a$ and $b$ are in $k^{p}$ and $n_{1} \geqq 1$ (since $\left.a_{r} \notin k^{p^{n}}\right)$. If $u \notin k$, then $u$ is transcendental over $k$, $a u^{\left(p^{r}-1\right) p^{n_{1}}}-b+v^{p^{n_{1}}}$ is irreducible in $k(u)[v]$, but becomes reducible upon adjoining $a^{p^{-1}}$ and $b^{p-1}$ to $k$. This shows that $k(u, v) \subset K$ is not separable, contradicting the separability of $K$. Hence $x_{0}=u^{-1} \in k$. Taking $(*)$ first with $i=$ $1, \cdots, n-1$, we see that $x_{i} \in k$, and then $y_{i-n} \in k$ follows for $i \geqq n$.

The proof above suggests examples showing that the assumptions in (ii) cannot be weakened. First, let $k=k_{0}(a, b)$ with $a, b$ algebraically independent over $k_{0}$. Then $G=(F, 1+a F)$ and $G^{\prime}=(F, 1+b F)$ are not isomorphic over $k$. On the other hand, we can define $K=k(u, v)$ by $a u^{p(p-1)}-b+v^{p}=0$. One checks that $k$ is algebraically closed in $K$. But now $1+b F=u^{-p}(1+a F) u^{p}+F v$, so that $G_{K} \cong G_{K}^{\prime}$. Next, suspose $k$ contains elements $a$ and $c$ such that $a \notin k^{p}$ and $c \notin k^{q-1}$ where $q=p^{m}>2$. Let $G=\left(F^{m}, 1+a F^{m}\right), G^{\prime}=\left(F^{m}, 1+c^{q} a F^{m}\right)$. If $K \supset k$, then $G_{K} \cong G_{K}^{\prime}$ if and only if $a u^{q(q-1)}+v^{q}=c^{q} a$ has a solution with $u$, $v \in K$. If $K$ is separable over $k$, then $a \notin K^{p}$, so necessarily $v=0$ and $u^{q-1}=c$. This is possible over a finite separable extension of $k$ but not over $k$. We will see below that this example is typical (cf. 3.1.1.).

3. Let $G$ and $G_{1}$ be forms of $\mathbf{G}_{a}$ written as fiber products

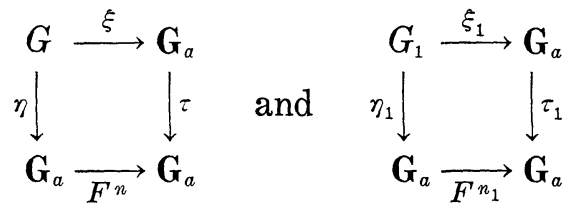

with $n=n(G)$ and $n_{1}=n\left(G_{1}\right)$ (cf. 1.4). Suppose $\psi \in \operatorname{Hom}_{k}\left(G, G_{1}\right)$ is nonzero. Then $\Theta^{n} \psi: \mathbf{G}_{a} \rightarrow \Theta^{n} G_{1}$ is nonzero, and since a nonzero homomorphic image of $\mathbf{G}_{a}$ is isomorphic with $\mathbf{G}_{a}$ (cf. [6], p. 101, lemma), we must have $n_{2}=n-n_{1} \geqq 0$. Now $F^{n_{2}} \xi_{1} \psi$ has inseparable degree $\geqq p^{n}$ and therefore factors through $\xi$. This gives a commutative diagram 


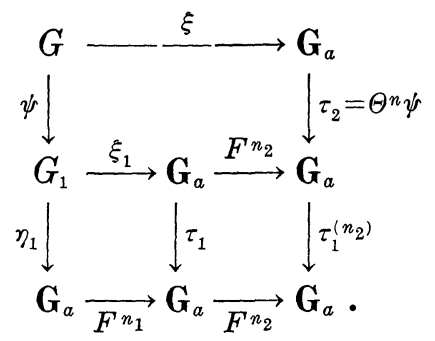

If $\psi$ is separable, so are $\tau_{2}$ and $\tau_{1}^{\left(n_{2}\right)} \tau_{2}$. This shows that one can use the big square to define $G$ as a fiber product, that is, $G \cong\left(F^{n}, \tau_{1}^{\left(n_{2}\right)} \tau_{2}\right)$. By 2.3 there exist $\rho \in A^{*}$ and $\sigma \in A$ such that

$$
\tau_{1}^{\left(n_{2}\right)} \tau_{2}=\rho^{(n)} \tau+F^{n} \sigma \text {. }
$$

(No $c$ appears since $\xi$ is left unchanged.) Conversely, if $\tau_{2}$ satisfies $(*)$, there is a unique $\psi$ making the diagram commutative. So separable homomorphisms $\psi: G \rightarrow G_{1}$ are in one-to-one correspondence with those $\tau_{2} \in A^{*}$ for which a solution to $(*)$ exists.

THeOREM 3.1. Let $G$ be a form of $\mathbf{G}_{a}, G \not \mathbf{G}_{a}$. Then $\operatorname{End}_{k} G$ may be identified with a finite subfield of $k$. If $\operatorname{End}_{k_{s}} G_{k_{s}}=\mathbf{F}_{q}$ and $k \subset K \subset k_{s}$, then $\operatorname{End}_{K} G_{K}=K \cap \mathbf{F}_{q}$.

Proof. Let $G=\left(F^{n}, \tau\right), n=n(G)$, and suppose $\psi: G \rightarrow G$ is nonzero. If $\psi$ is not separable, there is a nonzero homomorphism $\Theta G \rightarrow$ G. Since $n(\Theta G)<n(G)$, this is impossible, as we have seen. So $\psi$ is separable and $\tau_{2}=\Theta^{n} \psi$ satisfies a relation

$$
\tau \tau_{2}=\rho^{(n)} \tau+F^{n} \sigma, \quad \rho \in A^{*}, \sigma \in A .
$$

We will assume, as we may, that $\operatorname{deg} \rho<p^{n}$. Since $\Theta^{r}, r \geqq 0$, is a faithfully flat base change functor, $\Theta^{r}: \operatorname{End}_{k} G \rightarrow \operatorname{End}_{k} \Theta^{r} G$ is injective and moreover $\Theta^{r} \psi$ is a monomorphism (epimorphism) if and only if $\psi$ is. Taking $r=n-1$, we see that it is enough to prove the first statement in case $n=1$. We can then choose $\rho=a \in k^{*}$ and $\tau=1+$ $a_{1} F^{m_{1}}+\cdots+a_{s} F^{m_{s}}$ with $1 \leqq m_{1}<m_{2}<\cdots<m_{s}$ and $a_{i} \notin k^{p}$. Let $\tau_{2}=c_{0}+c_{1} F+\cdots+c_{r} F^{r}, c_{0} \neq 0$ and $c_{r} \neq 0$. Comparing coefficients in $(*)$, we get $a_{s} c_{r}^{p_{s}} \in k^{p}$ unless $r=0$. Since $m_{s} \geqq 1$ and $a_{s} \notin k^{p}$, we actually have $r=0$ and $\tau_{2}=c_{0}=c \in k^{*}$. (*) now reduces to $a^{p} \tau-$ $\tau c \in F A$, and this gives $a^{p}-c=0$ and $\left(c-c^{p^{m}}\right) a_{i} \in k^{p}, i=1, \cdots, s$. Since $a_{i} \notin k^{p}$, this implies $c-c^{p^{m_{i}}}=0$. Or, equivalently, $c-c^{p^{m}}=0$ where $m$ is the greatest common divisor of $m_{1}, \cdots, m_{s}$. Conversely $\tau c=c \tau$ for such $c$ and if $c \neq 0$, it lifts to an automorphism of $G$. Hence $\operatorname{End}_{k} G=k \cap \mathbf{F}_{p^{m}}$ in this case.

Now let $n \geqq 1, \mathbf{F}_{q}=\operatorname{End}_{k_{s}} G_{k_{s}}, k \subset K \subset k_{s}$ and $\tau_{2}=c \in K \cap \mathbf{F}_{q}^{*}$. To 
show that $c \in \operatorname{End}_{K} G_{K}$, we have to solve (*) with $\rho, \sigma \in A_{K}$. However there exists a solution over $k_{s}$, and applying to it a $K$-automorphism $\lambda$ of $k_{s}$, we get $\tau c=\lambda(\tau c)=\lambda\left(\rho^{(n)}\right)+F^{n} \lambda(\sigma)$ and $0=\left(\rho^{(n)}-\lambda\left(\rho^{(n)}\right)\right) \tau+$ $F^{n}(\sigma-\lambda(\sigma))$. Multiplying by $\tau^{-1}$ (in $\left.\hat{A}_{K}\right)$, we have $0=\left(\rho^{(n)}-\lambda\left(\rho^{(n)}\right)\right)+$ $F^{n}(\sigma-\lambda(\sigma)) \tau^{-1}$, giving $\rho^{(n)}=\lambda\left(\rho^{(n)}\right)$ and $\sigma=\lambda(\sigma)$ since $\operatorname{deg} \rho<p^{n}$. Hence $\rho, \sigma \in A_{K}$.

The theorem states that the automorphism functor of $G$ coincides with the functor $\mu_{r}$ ( $r$-th roots of unity, $r=q-1$ prime to $p$ ) on separable algebraic extensions of $k$. Galois cohomology therefore gives (for details we refer to [8], in particular I, $\S 5$, II, $\S 1$ and III, $\S 1$ ):

CoROLlary 3.1.1. Let $E\left(k_{s} / k, G\right)$ be the set of $k_{s} / k$-forms of $G$. Then $E\left(k_{s} / k, G\right)=H^{1}\left(k, \mathbf{F}_{q}^{*}\right) \cong k^{*} / k^{* q-1}$.

4. We turn now to forms of $\mathbf{A}^{1}$ that fail to be groups by just the absence of a rational point.

Proposition 4.1. Let $X$ be a form of $\mathbf{A}^{1}$ and suppose that $X_{k_{s}}$ admits a group structure. Then $X$ is a principal homogeneous space for a form $G$ of $\mathbf{G}_{a}$ determined uniquely by $X$. Moreover, $X=$ Spec $k[x, y] / I, G=\operatorname{Spec} k[u, v] / J$ where $I$ and $J$ are generated respectively by $y^{p^{n}}-b-f(x)$ and $v^{p^{n}}-f(u)$ with $b \in k$ and $f$ a separable p-polynomial. Conversely, if $X$ and $G$ are defined as above, then $X$ is a principal homogeneous space for $G$.

Proof. Let $X=\operatorname{Spec} R$. As in the proof of 2.1, we have $\left(k, \varphi^{n}\right) \bigotimes_{k} R \cong k[t]$ for some $n, t=\sum a_{i} \otimes y_{i}$ with $a_{i} \in k$ linearly independent over $k^{p^{n}}$, and $y_{i}^{p^{n}}=g_{i}(x) \in k[x]$ with $x=F_{R}^{n}(t)$. Let $q \in X_{k_{s}}$ be rational over $k_{s}$ and let $c_{i} \in k_{s}$ be the residue of $y_{i}$ at $q$. Put $y_{i}^{\prime}=$ $y_{i}-c_{i}, t^{\prime}=t-\sum a_{i} c_{i}^{p^{n}}=t-c$ and $x^{\prime}=x-c$. Then $t^{\prime}=\sum a_{i} \otimes y_{i}^{\prime}$, $q$ lies above the point $t^{\prime}=0$ of $\mathbf{A}_{k_{s}}^{1} \cong \Theta^{n} X_{k_{s}}$ and we can choose $q$ as the origin of the group structure supposed to exist on $X_{k_{s}}$. The $a_{i}$ remain linearly independent over $k_{s}^{p^{n}}$ and we have $y^{\prime p^{n}}=f_{i}\left(x^{\prime}\right)$ with $f_{i}$ a $p$-polynomial as in the proof of 2.1. Hence $g_{i}(x)=y_{i}^{p^{n}}=b_{i}+f_{i}(x)$ with $b_{i}=c_{i}^{p^{n}}-f_{i}(c)$, and $g_{i}(x) \in k[x]$ implies $b_{i} \in k$ and $f_{i}(x) \in k[x]$. If $y$ is a separating variable for $\kappa(X)$ picked from the $y_{i}$, we get $y^{p^{n}}=$ $b+f(x)$ where $f$ has nonzero linear term. As before, this implies $R=$ $k[x, y]$. Let $G=\operatorname{Spec} S, S=k[u, v]$ with $v^{p^{n}}=f(u)$. Then $\alpha: R \rightarrow$ $R \otimes_{k} S, \alpha(x)=x \otimes 1+1 \otimes u$ and $\alpha(y)=y \otimes 1+1 \otimes v$, defines an action of $G$ on $X . \quad \bar{\alpha}: R \otimes_{k} R \rightarrow R \otimes_{k} S$ defined by $\bar{\alpha}(w \otimes z)=$ $(w \otimes 1) \alpha(z)$ is an isomorphism and gives an isomorphism (over $X$ ) $G \times_{k} X \stackrel{\longrightarrow}{\longrightarrow} \times_{k} X$. Hence $X$ is a principal homogeneous space for $G$. If this is also true for $G_{1}$, we get an isomorphism (over $X$ ) $G \times{ }_{k} X \simeq G_{1} \times_{k} X$. Applying 2.6 (ii) to the fiber over the generic 
point of $X$, we see that $G \cong G_{1}$.

Principal homogeneous spaces for $G$ are clasified by $H^{1}(k, G)$ (cf. [8], I, Proposition 33). Let $G=\left(F^{n}, \tau\right)$. Then there is a commutative diagram with exact rows:

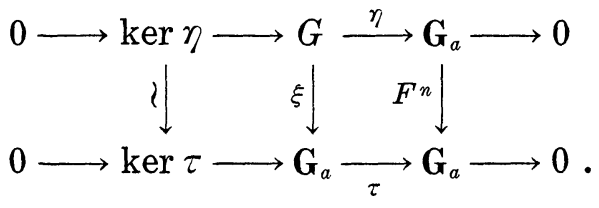

The exact cohomology sequence and $H^{1}\left(k, \mathbf{G}_{a}\right)=0$ give $H^{1}(k, G)=$ $k / f(k)+k^{p^{n}}$, where $f$ is the $p$-polynomial corresponding to $\tau$. The Galois group of the spliting field of $0=b+f(x)=b+a_{0} x+\cdots+a_{m} x^{p^{m}}$, $a_{0} \neq 0$, is isomorphic to a subgroup of $f^{-1}(0) \subset k_{s}$. Hence $f(k)=k$ if $k$ has no normal extension of degree $p$, and $H^{1}(k, G)=0$ for all forms $G$ of $\mathbf{G}_{a}$ in that case. The author does not know whether the converse of this statement is true if $k$ is not perfect.

In [4] Rosenlicht characterized curves that are "exceptional" in the sense that the genus $g$ is $\geqq 1$ and the group of automorphisms (leaving a point fixed if $g=1$ ) is infinite. We give another characterization, already implicit in [4], p. 10, theorem, assuming the exceptional case over $k_{s}$ only.

THeorem 4.2. Let $P$ be a complete regular curve such that $P_{k_{s}}$ is exceptional. Then $P$ has exactly one singular point $q, q$ is purely inseparable over $k$, and $X=P-\{q\}$ is a principal homogeneous space for a form of $\mathbf{G}_{a}$.

Proof. It is enough to prove the first statement in case $k=k_{s}$. It is then taken directly from [4], p. 5, lemma. It is also shown there that $\widetilde{P}_{k_{i}}$ has genus zero. Hence $X=P-\{q\}$ is a form of $\mathbf{A}^{1}$ and we have $F_{X}^{n}: X \rightarrow \Theta^{n} X \cong \mathbf{A}^{1}=$ Spec $k[t]$ for some $n$. This gives an injection $\Theta^{n}: \operatorname{Aut}_{k} X \rightarrow \operatorname{Aut}_{k} \mathbf{A}^{1}$. Now let $k=k_{s}$. It then follows from [4], loc. cit., that $\mathrm{Aut}_{k} X$ has an infinite subset of automorphisms operating without fixed point. Hence $\Theta^{n}\left(\mathrm{Aut}_{k} X\right)$ contains infinitely many translations $t \mapsto t+b$. With notations as in the proof of 4.1, write $t=\sum a_{i} \otimes y_{i}, 1 \otimes y_{i}=f_{i}(t)$, with $t$ so chosen that the point $q_{0} \in X$ above $t=0$ is rational. If $c_{i}$ is the residue of $y_{i}$ at $q_{0}$, we have $f_{i}(0)=c_{i}^{p^{n}} \in k^{p^{n}}$. Since $0=\sum a_{i} f_{i}(0)$, we get $f_{i}(0)=0$. If $T_{b}$ is the automorphism of $X$ inducing $t \mapsto t+b$, we have $t+b=\sum a_{i} \otimes T_{b}^{*}\left(y_{i}\right)$. Let $b_{i} \in k$ be the residue of $y_{i}$ at $T_{b}\left(q_{0}\right)$. Then $b=\sum a_{i} b_{i}^{p^{n}}$ and $t+b=$ $\sum a_{i} \otimes\left(y_{i}+b_{i}\right)$. Hence $T_{b}^{*}\left(y_{i}\right)=y_{i}+b_{i}$ and $f_{i}(t+b)=1 \otimes T_{b}^{*}\left(y_{i}\right)=$ $f_{i}(t)+b_{i}^{p^{n}}$. With $t=0$, this shows $b_{i}^{p^{n}}=f_{i}(b)$. Since this holds for infinitely many $b$, each $f_{i}$ is a $p$-polynomial. Hene $X$ has a group 
structure (over $k_{s}$ ) and 4.1 applies.

If $X$ is a principal homogeneous space for a form $G$ of $\mathbf{G}_{a}$ and $P \supset X$ a complete regular curve, then $G\left(k_{s}\right) \subset \mathrm{Aut}_{k_{s}} P_{k_{s}}$ is infinite. So $P_{k_{s}}$ is exceptional if the genus $g$ of $P$ is positive. The cases $g=0$ as well as $g=1$ can be settled completely. Excluding the trivial case $X=\mathbf{A}^{1}$, we have: If $g=0$, then char $k=2$. If $g=1$, then char $k=3$. Moreover, $X=\operatorname{Spec} k[\mathrm{x}, y] / I$ where $I$ is generated by $y^{p}-b-x-a x^{p}$ with $p=2$ or 3 respectively and $a, b \in k$.

It is enough to prove the corresponding statement for the groups $G$ that are involved, that is, we may assume $X=G$ has a rational point. Now, by a theorem of Tate ([9], Corollary 2), the genus changes by a multiple of $1 / 2(p-1)$ on passage from $X$ to $\theta X$. On the other hand, if $O$ is the local ring of $P-X$, the genus change is $\operatorname{dim}_{k} O_{1} / O^{\prime}$ where $\mathscr{O}^{\prime}=(k, \varphi) \otimes_{k} \bigcirc$ and $\mathscr{O}_{1}$ is the normalization of $\mathscr{O}^{\prime}$ (cf. [7], p. 73, example). So a drop in genus occurs unless $\mathcal{O}$ is nonsingular. But then $P$ is nonsingular, so $g=0$ and $P \cong \mathbf{P}^{1}$. Excluding the case $G=\mathbf{G}_{a}$ we must have $\mathbf{P}^{1}-G$ of degree 2 (cf. [5], p. 35 or the remark in the introduction). Hence $p=2$ and $n(G)=1$. If $p>2$, we see that $g \geqq 1 / 2 n(G)(p-1)$. So $g=1$ implies $n(G)=1$ and $p=3$. In both cases $(g=0$ or 1$) G=\operatorname{Spec} k[x, y]$ with $y^{p}=x+a_{1} x^{p}+\cdots+a_{m} x^{p^{m}}$ and $a_{m} \notin k^{p} \quad$ (cf. 2.1). Using [9], proposition, one checks that then $g=$ $1 / 2(p-1)\left(p^{m}-2\right)$. So necessarily $m=1$.

\section{REFERENCES}

1. C. Chevalley, Introduction to the theory of algebraic functions of one variable, Math. Surv. VI, New York, 1951.

2. N. Jacobson, Lectures in abstract algebra, vol. III, D. Van Nostrand Co., Princeton, 1964.

3. F. Oort, Commutative group schemes, Lecture Notes in Mathematics 15, Springer, Berlin, 1965.

4. M. Rosenlicht, Automorphisms of function fields, Trans. Amer. Math. Soc. 79 (1955), 1-11.

5. - Some rationality questions on algebraic groups, Annali di Mat. (IV) 43 (1957), 25-50.

6. - Questions of rationality for solvable algebraic groups over nonperfect fields, Annali di mat., (IV) 61 (1963), 97-120.

7. J. P. Serre, Groupes Algébriques et Corps de Classes, Hermann, Paris, 1959.

8. Cohomologie Galoisienne, Lecture Notes in Mathematics 5, Springer, Berlin, 1964.

9. J. Tate, Genus change in inseparable extensions of function fields, Proc. Amer. Math. Soc. 3 (1952), 400-406.

Received May 16, 1969. This research was supported in part by the U. S. Army Research Office (Durham).

HARVARD UNIVERSITY 



\section{PACIFIC JOURNAL OF MATHEMATICS}

\section{EDITORS}

\author{
H. SAMELSON \\ Stanford University \\ Stanford, California 94305 \\ RICHARD PIERCE \\ University of Washington \\ Seattle, Washington 98105
}

J. DugundJI

Department of Mathematics University of Southern California Los Angeles, California 90007

BASIL GORDON*

University of California

Los Angeles, California 90024

\section{ASSOCIATE EDITORS}
E. F. BECKENBACH
B. H. NEUMANN
F. WOLE
K. YOSHIDA

\section{SUPPORTING INSTITUTIONS}

\author{
UNIVERSITY OF BRITISH COLUMBIA \\ CALIFORNIA INSTITUTE OF TECHNOLOGY \\ UNIVERSITY OF CALIFORNIA \\ MONTANA STATE UNIVERSITY \\ UNIVERSITY OF NEVADA \\ NEW MEXICO STATE UNIVERSITY \\ OREGON STATE UNIVERSITY \\ UNIVERSITY OF OREGON \\ OSAKA UNIVERSITY \\ UNIVERSITY OF SOUTHERN CALIFORNIA
}

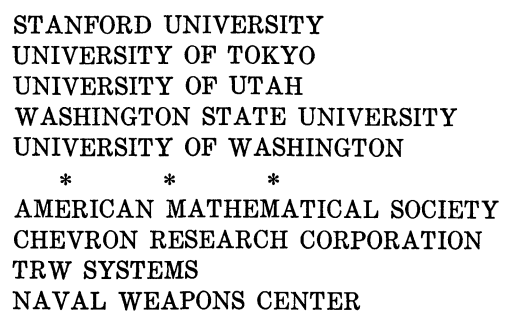

The Supporting Institutions listed above contribute to the cost of publication of this Journal, but they are not owners or publishers and have no responsibility for its content or policies.

Mathematical papers intended for publication in the Pacific Journal of Mathematics should be in typed form or offset-reproduced, (not dittoed), double spaced with large margins. Underline Greek letters in red, German in green, and script in blue. The first paragraph or two must be capable of being used separately as a synopsis of the entire paper. The editorial "we" must not be used in the synopsis, and items of the bibliography should not be cited there unless absolutely necessary, in which case they must be identified by author and Journal, rather than by item number. Manuscripts, in duplicate if possible, may be sent to any one of the four editors. Please classify according to the scheme of Math. Rev. 36, 1539-1546. All other communications to the editors should be addressed to the managing editor, Richard Arens, University of California, Los Angeles, California, 90024.

50 reprints are provided free for each article; additional copies may be obtained at cost in multiples of 50 .

The Pacific Journal of Mathematics is published monthly. Effective with Volume 16 the price per volume (3 numbers) is $\$ 8.00$; single issues, $\$ 3.00$. Special price for current issues to individual faculty members of supporting institutions and to individual members of the American Mathematical Society: $\$ 4.00$ per volume; single issues $\$ 1.50$. Back numbers are available.

Subscriptions, orders for back numbers, and changes of address should be sent to Pacific Journal of Mathematics, 103 Highland Boulevard, Berkeley, California, 94708.

PUBLISHED BY PACIFIC JOURNAL OF MATHEMATICS, A NON-PROFIT CORPORATION

Printed at Kokusai Bunken Insatsusha (International Academic Printing Co., Ltd.), 7-17, Fujimi 2-chome, Chiyoda-ku, Tokyo, Japan.

* Acting Managing Editor. 


\section{Pacific Journal of Mathematics}

\section{Vol. 32, No. $2 \quad$ February, 1970}

Harry P. Allen and Joseph Cooley Ferrar, Jordan algebras and exceptional subalgebras of the exceptional algebra $E_{6} \ldots \ldots \ldots \ldots \ldots \ldots \ldots 283$

David Wilmot Barnette and Branko Grünbaum, Preassigning the shape of a

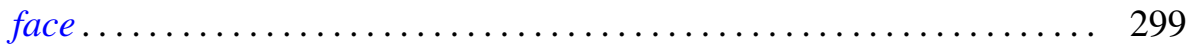

Robert Francis Craggs, Involutions of the 3-sphere which fix 2-spheres . . . . 307

David William Dean, Bor-Luh Lin and Ivan Singer, On k-shrinking and $k$-boundedly complete bases in Banach spaces ................ 323

Martin Engert, Finite dimensional translation invariant subspaces ....... 333

Kenneth Lewis Fields, On the global dimension of residue rings ......... 345

Howard Gorman, The Brandt condition and invertibility of modules ....... 351

Benjamin Rigler Halpern, A characterization of the circle and interval ..... 373

Albert Emerson Hurd, A uniqueness theorem for second order quasilinear

hyperbolic equations ............................... 415

James Frederick Hurley, Composition series in Chevalley algebras ...... 429

Meira Lavie, Disconjugacy of linear differential equations in the complex

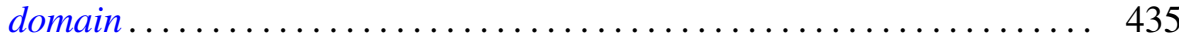

Jimmie Don Lawson, Lattices with no interval homomorphisms ......... 459

Roger McCann, A classification of center-foci ................. 467

Evelyn Rupard McMillan, On continuity conditions for functions . . . . . . . 479

Graciano de Oliveira, A conjecture and some problems on permanents .... 495

David L. Parrott and S. K. Wong, On the Higman-Sims simple group of order $44,352,000$.

Jerome L. Paul, Extending homeomorphisms ................. 517

Thomas Benny Rushing, Unknotting unions of cells .............. 521

Peter Russell, Forms of the affine line and its additive group.......... 527

Niel Shilkret, Non-Archimedean Gelfand theory ................. 541

Alfred Esperanza Tong, Diagonal submatrices of matrix maps.......... 551 